\title{
1 Monitoring of microalgal cultivations with on-line, flow-through microscopy
}

2

3 Ivo Havlik ${ }^{* 1}$, Kenneth F. Reardon ${ }^{2}$, Mehmet Ünal ${ }^{1}$, Patrick Lindner ${ }^{1}$, Andreas Prediger ${ }^{1}$, $4 \quad$ Alexander Babitzky ${ }^{1}$, Sascha Beutel ${ }^{1}$, and Thomas Scheper ${ }^{1}$

5

$6{ }^{1}$ Leibniz University Hannover, Institute of Technical Chemistry, Callinstrasse 5, 30167

7 Hannover, Germany

$8 \quad{ }^{2}$ Department of Chemical and Biological Engineering, Colorado State University, Fort

9 Collins, CO 80523-1370, USA

10

11

$12{ }^{*}$ Corresponding author: Ivo Havlik

13 Phone: +49-511-762-2569

14 Email: Havlik@iftc.uni-hannover.de

15

16 


\section{Abstract}

18 Microalgal cultivations present challenges for monitoring and process control posed by their

19 large scale and the likelihood that they will be composed of multiple species. Cell concentration is a fundamental parameter in any cultivation but is typically performed using off-line methods that may be time-consuming, laborious, or subject to interferences. Here, an in-situ microscope has been adapted to monitoring microalgal cultivations by adding a flow-

23 through cell and adjusting image-processing algorithms. After installation in the bypass of a

24 photobioreactor, the microscope enabled the continuous, automated acquisition of cell

25 count, cell size, and cell morphology data on-line during cultivation processes over a period

26 of 20 days, without sampling. The flow-through microscope was tested in cultivations of

27 Chlamydomonas reinhardtii and Chlorella vulgaris. Cell concentration measurements were in agreement with off-line optical density measurements for both species. In addition, cell size and morphology distributions were obtained that revealed population shifts during the

30 cultivation of $C$. vulgaris. This monitoring system thus provides a means to obtain detailed,

31 non-invasive insights of microalgal cultivation processes.

34 Keywords

35 Flow-through microscopy, microalgae, automated image processing, cell count, cell size 36 distribution 
39 The industrial cultivation of microalgae has been the focus of increased attention in recent

40 years, although the first commercial operations date to the 1960s. Various microalgae are

41 cultivated to produce food, food supplements, pigments, and lipids for conversion to biofuels.

42 Microalgae are usually cultivated in batch or semi-batch processes with cultivation times of up to 20 days. The monitoring of microalgal cultivations is of importance for process control.

Of particular interest are parameters related to the biological system, including cell concentration and composition, which provide information about the status of the cultivation.

Data on cell morphology and size are useful for monitoring the presence of contaminating species and the health of the culture. Ideally, these measurements would be made continuously, with the sensor system interfaced with the photobioreactor or pond in either an in-situ or an on-line format [1].

One approach to this goal is the use of continuous, non-invasive microscopic monitoring. The first in-situ microscope (ISM) was developed in 1990 [2] and has since been improved by several researchers [3]. It has been employed for the in-situ monitoring of yeast, mammalian, and microcarrier cultivations as well as crystallization processes of amino acids, proteins, and pharmaceuticals [4]. Most ISM systems are based on a transmitted light microscope and can be mounted in a $25-\mathrm{mm}$ side port of a bioreactor. The sampling zone is thus immersed in the cultivation medium. Images of microorganisms or crystals are acquired and processed using particle-specific algorithms, yielding estimates of several parameters, including particle count, size and morphology.

60

The goal of this project was to develop and evaluate the ISM strategy for monitoring microalgal cultivations. Cultivations of two algal species were conducted and compared with standard off-line measurements of optical density.

64 


\section{Materials and Methods}

\subsection{Flow-through microscope hardware}

The flow-through microscope (FTM) is a modified version of the in-situ microscope ISM III XTF (Sartorius Stedim Biotech GmbH, Göttingen, Germany) [5]. It has been used successfully for monitoring of various biotechnological processes such as cultivations of microorganisms and protein crystallization. The ISM III XTF is a transmitted light, bright-field microscope that can be mounted in a $25-\mathrm{mm}$ side port of a bioreactor.

For the monitoring of microalgal cultivations, several modifications of the ISM III XTF were necessary to allow removal of adhered cells within the measuring zone, provide a replaceable light source, and integrate the device into the glass tubular photobioreactor used in this study. To accomplish these goals, the outer tube of the ISM was redesigned by replacing the sampling zone with a flow cell and by adding inlet and outlet metal tubes that can be integrated into the bypass of a photobioreactor. The upper segment of the microscope containing camera, objective lens, and motors was mounted on the outer tube (Fig. 1). Since the whole system has a modular construction, individual parts could be replaced easily. A white LED was attached on the other side of the flow cell (Fig. 1). This construction allowed the flushing of the microscope windows by temporarily increasing the flow rate through the flow cell.

\subsection{Image analysis and hardware control software}

It was also necessary to alter the image-processing algorithms of the ISM III XTF to recognize microalgal cells. The original instrument software includes the control program InSitu Control for controlling the ISM hardware and camera parameters and for recording microscope images, as well as the image processing software InSitu Analysis for performing cell recognition and to allow parameter computation either on- or off-line. The algorithms for image processing for cell detection were originally designed for yeast cells and were based on border-tracking methods. Differences of grey values to the mode value (image 
background) were used to define the border of possible cell objects. Since algae images tended to be noisier, and consequently produced blurry object borders, this led to processing artifacts in this application to algal cells.

Modification of the software consisted of migration from Delphi to C\#, and implementing the SUSAN procedure [6] for border tracking and cell detection to allow better object recognition from noisy and out-of-focus images. Furthermore, the "Cell Wiper" function was introduced to enable recognition of cells that have stuck to the sampling zone surface. These adhered cells could be then excluded from counting. For each microalgal strain, additional strainspecific algorithm optimization was necessary to account for different cell shapes.

The resulting software is capable of computing three process variables as primary information: cell count in an image, and cell size and cell eccentricity for each cell identified in the image. Furthermore, the software produces other information from these primary data: cell volume of individual cells, total cell volume in a given liquid volume (biomass concentration), detection of double cells and cell clusters, and classification into large, medium, and small cells. Processing parameters and all results for each image are recorded in a separate file linked to the image. From these files, all data can be exported, for each cell and each image individually or as a summary, into a .csv file. Cell size distributions can also be exported as a histogram. All variables are visualized in the GUI in real time.

\subsection{Microscope calibration}

For absolute cell area determination, the microscope-camera system was calibrated. A film with a microscale was inserted into the measuring zone and pixels over a distance were counted manually in the image. The area of one pixel was computed from pixel count per micrometer. When the 10X microscope objective was used, a pixel had an area of $0.67 \mu \mathrm{m}^{2}$, while the 20X objective yielded a conversion of $0.17 \mu \mathrm{m}^{2}$ per pixel. 


\subsection{Photobioreactor system}

123 Two photobioreactor (PBR) cultivation systems were used for testing the FTM. Both are

124 based on glass tubes of $45-\mathrm{cm}$ length and outside diameter of $8 \mathrm{~cm}$ (volume $1.9 \mathrm{~L}$ ) equipped

125 with two tubing connections and an inner glass tube of $2.4-\mathrm{cm}$ inside diameter placed along

126 the centerline. The light source was an Osram $64013 \mathrm{~W}$ Universal White fluorescent lamp

127 that was located in the inner glass tube and provided illumination with average intensity

128 (PAR) of $58 \mu \mathrm{mol} \mathrm{s}{ }^{-1} \mathrm{~m}^{-2}$ as measured $3 \mathrm{~cm}$ from the lamp surface. To accommodate the

129 aeration, cooling, and sensors, two variants of a monitoring/addition vessel (MAV) were

130 employed, both with a working volume of about $0.5 \mathrm{~L}$. The first MAV was a glass vessel

131 equipped with sensor ports in the lid for temperature, $\mathrm{pH}$, and $\mathrm{pO}_{2}$, and with a gas inlet and

132 outlet. The second MAV was a steel double-jacketed unit with an inoculation port, a mixer,

133 ports for temperature and $\mathrm{pH}$ sensors, and connections for gas inlet and outlet. In each

134 experiment, one of these MAVs was attached to the glass tube by flexible tubing, and a

135 peristaltic pump was used to circulate the growth medium between the glass tube and the

136 MAV. Gas sparging took place only in the MAV. The first system, PBR-1, consisted of two

137 glass tubes with the glass MAV and had a total volume of $4.4 \mathrm{~L}$, while PBR-2 consisted of a

138 single glass tube and the stainless steel MAV; this system had a total volume of $2.4 \mathrm{~L}$. In

139 PBR-1, temperature and $\mathrm{pH}$ were measured but not controlled, whereas temperature and $\mathrm{pH}$

140 were controlled by a Sartorius control unit Biostat B in PBR-2. In all experiments, $\mathrm{CO}_{2}$ was

141 supplied by sparging the liquid in the MAV with a mixture of $3 \% \mathrm{CO}_{2}$ in air at $1 \mathrm{vvm}$. The

142 FTM was placed in the PBR bypass and supplied with cell suspension using a second

143 peristaltic pump (Fig. 2).

\subsection{Algal cultures, experimental conditions, and measurements}

146 For all cultivation experiments, axenic cultures under sterile conditions were employed. TAP

147 medium [7] was used for all cultivations. On-line cell measurements using the flow-through

148 microscope were evaluated with the modified InSitu Control and InSitu Analysis software. 
150 A set of four cultivations of Chlamydomonas reinhardtii (strain SAG 33.89, SAG Culture

151 Collection, Göttingen, Germany) was performed in the PBR-1 system at approximately $26^{\circ} \mathrm{C}$

152 and $\mathrm{pH}$ 7. C. reinhardtii is a green microalga with approximately spherical shape and a

153 diameter of 14 to $22 \mu \mathrm{m}$. Cultivations were inoculated with a $100-\mathrm{mL}$ culture of C. reinhardtii

154 cells grown for 10 days in an illuminated shake flask. The optical density (OD) during the

155 cultivation experiments was measured off-line at $550 \mathrm{~nm}$ approximately every $24 \mathrm{~h}$ using a

156 Uvikon spectrophotometer. A 10X objective was used in the flow-through microscope. The

157 on-line cell count was computed as cell count/image. Image acquisition and evaluation was

158 performed in cycles every hour, each cycle comprising 100 images in 1-s interval.

160 A second set of two cultivations was carried out using Chlorella vulgaris, a green microalga 161 with spherical shape and a diameter of 4 to $10 \mu \mathrm{m}$. The cultivation was performed in the 162 PBR-2 system at $26^{\circ} \mathrm{C}$ and $\mathrm{pH}$ 7, inoculated with a 100-mL culture of $C$. vulgaris cells grown 163 for 10 days in an illuminated shake flask. The optical density was measured at $750 \mathrm{~nm}$, 164 outside the absorption range of both chlorophyll a and chlorophyll b, to avoid interference by 165 variable chlorophyll content. As Chlorella cells are smaller, a 20X objective was used in the 166 flow-through microscope. The on-line cell count was measured as in the C. reinhardtii 167 cultivation and computed also as cell count/image.

\section{Results and Discussion}

\subsection{C. reinhardtii cultivation monitoring}

172 For the initial evaluation of the FTM for algal cultivation monitoring, cell number concentrations were computed from the analysis of images acquired by the FTM during the C. reinhardtii cultivations. Figure 3 is a comparison of these FTM-derived data with the off-

175 line OD measurements at $550 \mathrm{~nm}$. Although the data computed from the images of the flow-

176 through microscope became noisier after $300 \mathrm{~h}$ of cultivation, filtering using a 12-h

177 asymmetric median was successful in providing a smoother output. Another comparison of 
178 the on-line FTM data and the off-line OD measurements was the calculation of the ratio of

179 the off-line optical density and the median of the cell count obtained from the flow-through

180 microscope data. Ideally, this ratio should be constant during the cultivation no matter the

181 dimensioning of both variables. In these measurements, the relative difference between the

182 two methods decreased with increasing cell concentration (Supplemental Data Figure S-1).

\subsection{C. vulgaris cultivation monitoring}

185 Based on experiences gained during the analysis of the first set of experiments, the data

186 collected by the FTM during the $C$. vulgaris cultivations were analyzed using several

187 additional methods. To account for the presence of a small number of clusters of cells that

188 might skew the data, information from the cell size and eccentricity data collected by the

189 FTM were used to create a function for elimination of likely cell clusters. FTM images from

190 the early growth phase were manually evaluated to determine the parameters of this

191 function, which is shown in Supplemental Data Figure S-2. Cell clusters were screened as

192 follows: (a) all objects larger than 1400 pixels were assumed to be clusters, (b) all objects

193 smaller than 600 pixels were assumed to be single cells, and (c) for objects in the size range

$194600-1400$ pixels, objects below the corresponding critical eccentricity for a given pixel size

195 (Supplemental Data Figure S-2) were assumed to be single cells. This screening process

196 resulted in the elimination of $3.6 \%$ of the images in the early and late growth phases, and $4 \%$

197 of the images in the stationary phase. These procedures yielded a dataset containing all

198 cells identified in the images acquired during the whole cultivation, with every cell linked to

199 its size and eccentricity, with cell clusters eliminated. All subsequent analyses resulting in

200 cell size and eccentricity distributions were carried out using individual cells and their

201 individual parameters.

203 These corrected, individual cell data were used to determine cell number concentrations in

204 1-h cycles, and these 1-h data were smoothed using a 12-h asymmetric moving average

205 filter to compare them to the off-line OD measurements (Fig. 4). As with the C. reinhardtii 
cultivations, the on-line data were in good agreement with the off-line OD measurements,

207 although the FTM values were somewhat lower near the start of the experiment (until about 165 h). Three growth phases were delineated: early growth (94-179 h), late growth (180$263 \mathrm{~h})$, and stationary (264-335 h).

211 Cell size distributions were computed from the corrected FTM data separately for each of the

212 three growth phases (Figure 5). A substantial shift in the size distribution between the early

213 growth and the later two stages can be observed by comparing average and median cell

214 sizes for each stage, computed from the dataset containing all cells detected in FTM images.

215 Average cell sizes in the early, late and stationary phases were 77,65 and $65 \mu \mathrm{m}^{2}$,

216 respectively, and median cell sizes were 71,61 and $62 \mu \mathrm{m}^{2}$, respectively. Specifically, the

217 cell population became smaller on average after the first $180 \mathrm{~h}$ because of the loss of larger

$218\left(90-160 \mu \mathrm{m}^{2}\right)$ cells. Distributions of cell size in bar chart format are shown in Supplemental

219 Data Figure S-3. Using off-line measurements in a Coulter Counter, Work et al. [8]

220 determined that $C$. reinhardtii CC124 cells grown in nitrogen-replete medium (corresponding

221 to the early growth phase) were $13 \%$ larger than cells grown in nitrogen-deficient medium

222 (corresponding to late growth and stationary phases). This shift in size distribution could explain the differences between the OD and FTM measurements between 100 and $200 \mathrm{~h}$.

Similarly, distributions of the cell morphology, expressed as eccentricity, were obtained from the $C$. vulgaris dataset, resulting from the analysis of FTM images corrected for presence of clusters. Distributions of eccentricity data in each of the three cultivation phases are shown in Figure 6, and distributions of eccentricity in bar chart format are shown in Supplemental Data Figure S-4. During the early growth phase, the $C$. vulgaris cells were less elongated than those later in the cultivation.

233 Although microalgal cell concentration data can be obtained off-line using flow cytometry, 
234 Coulter Counters, and optical absorbance, the results presented here are the first reports of

235 on-line, automated, continuous measurement of microalgal cell concentration. While other

236 methods such as optical absorbance can be modified for on-line application, the need to

237 provide dilution at higher cell concentrations, to avoid wavelengths affected by pigments,

238 and to correct for changes in cell size and composition (e.g., the presence of lipid bodies)

239 has proven challenging. Using FTM, these issues can be avoided, and additional

240 measurements such as the distributions of cell size and cell shape can be obtained.

241 Although shear stress from pumping the cells through the bypass may be a concern for

242 some microalgae, there was no discernible impact for the two species used in these

243 experiments.

245 Continuous measurements of cell concentration and cell size distribution have many

246 potential uses in large-scale microalgal cultivations. Nutrient addition and harvesting

247 strategies could be triggered when a particular cell concentration is reached, corrective

248 actions could be taken if the growth rate is not as expected, or the impact of predators (e.g.,

249 rotifers) could be detected at an early and potentially correctable stage. The additional

250 information content of continuous cell size distribution measurements could also provide

251 evidence of lipid accumulation (if associated with cell size increase) and the presence of

252 non-target microbial species. Those goals would be aided by modification of the image-

253 analysis software to provide data on the population morphology.

255 The modifications to the commercial ISM described here allow the imaging zone to be

256 cleaned automatically in the event of moderate cell adhesion. If more severe cell

257 accumulation is encountered, the microscope can easily be disconnected, cleaned, and

258 connected again under sterile conditions. Moreover, different light sources can be used with

259 simple replacement. 
261 The results reported here demonstrate the basic capabilities of this system for obtaining on-

262 line data during algal cultivations. Such a system can enable the collection of a high density

263 of information about cultivations in laboratory and commercial systems, potentially leading to

264 new insights into the basic and applied biology of algae.

\section{References}

[1] T. Scheper, B. Hitzmann, E. Stark, R. Ulber, R. Faurie, P. Sosnitza, K.F. Reardon, Bioanalytics: detailed insight into bioprocesses, Analytica Chimica Acta 400 (1999) 121134.

[2] H. Suhr, P. Speil, G. Wehnert, W. Storhas, In situ-Mikroskopiesonde und Messverfahren, Germany, 1990.

[3] T. Hopfner, A. Bluma, G. Rudolph, P. Lindner, T. Scheper, A review of non-invasive optical-based image analysis systems for continuous bioprocess monitoring, Bioprocess and Biosystems Engineering 33 (2010) 247-256.

[4] A. Bluma, T. Hopfner, A. Prediger, A. Glindkamp, S. Beutel, T. Scheper, Process analytical sensors and image-based techniques for single-use bioreactors, Engineering in Life Sciences 11 (2011) 550-553.

[5] G. Rudolph, P. Lindner, A. Gierse, A. Bluma, G. Martinez, B. Hitzmann, T. Scheper, Online monitoring of microcarrier based fibroblast cultivations with in situ microscopy, Biotechnology and Bioengineering 99 (2008) 136-145.

[6] S.M. Smith, J.M. Brady, SUSAN - A new approach to low level image processing, International Journal of Computer Vision 23 (1997) 45-78.

[7] E.H. Harris, The Chlamydomonas Sourcebook: Introduction to Chlamydomonas and Its Laboratory Use, 2nd Edition, Academic Press, 2008.

[8] V.H. Work, R. Radakovits, R.E. Jinkerson, J.E. Meuser, L.G. Elliott, D.J. Vinyard, L.M.L. Laurens, G.C. Dismukes, M.C. Posewitz, Increased Lipid Accumulation in the 
Figure captions

Figure 1. Schematic of the flow-through microscope. Inset: detail of measuring zone.

297 Figure 2. Schematic of the flow-through microscope placement in the photobioreactor bypass.

Figure 3. Comparison of off-line cell density measurements (OD) with data computed from in-situ FTM analysis during a cultivation of Chlamydomonas reinhardtii. Raw data were

Figure 4. Comparison of off-line cell density measurements $\left(\mathrm{OD}_{750}\right)$ with data computed from in-situ FTM analysis during a cultivation of Chlorella vulgaris. Cell count data were acquired and computed every hour, and were smoothed by a $12 \mathrm{~h}$ asymmetric moving

Figure 5. Cell size distributions in different phases of a C. vulgaris cultivation in PBR-2 as measured by the FTM. Phase 1 is the early growth phase (94-179 h), Phase 2 is the late growth phase (180-263 h), and Phase 3 is the stationary phase (264-335 h). Data

315 Figure 6. Distributions of $C$. vulgaris morphology in terms of eccentricity during different 316 phases of a $C$. vulgaris cultivation in PBR-2 as measured by the FTM. Eccentricity is 317 defined such that a value of 1.0 corresponds to a circle and larger values are ellipses. 318 Phase 1 is the early growth phase $(94-179$ h), Phase 2 is the late growth phase (180$263 \mathrm{~h})$, and Phase 3 is the stationary phase (264-335 h). Data resulting from cell clusters were removed using the experimentally derived function described in the text. 
1 Monitoring of microalgal cultivations with on-line, flow-through microscopy

2

3 Ivo Havlik ${ }^{* 1}$, Kenneth F. Reardon ${ }^{2}$, Mehmet Ünal ${ }^{1}$, Patrick Lindner ${ }^{1}$, Andreas Prediger ${ }^{1}$,

4 Alexander Babitzky ${ }^{1}$, Sascha Beutel ${ }^{1}$, and Thomas Scheper ${ }^{1}$

5

6

7 Acknowledgments

8 This work was supported by the Sustainable Bioenergy Development Center of Colorado

9 State University and by funds from the Jud and Pat Harper Professorship in Chemical and

10 Biological Engineering (to KFR).

11

12 

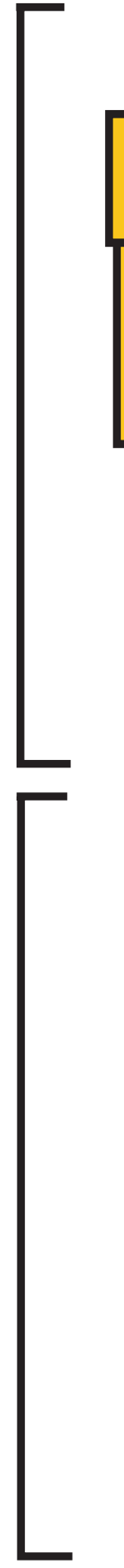

power supply

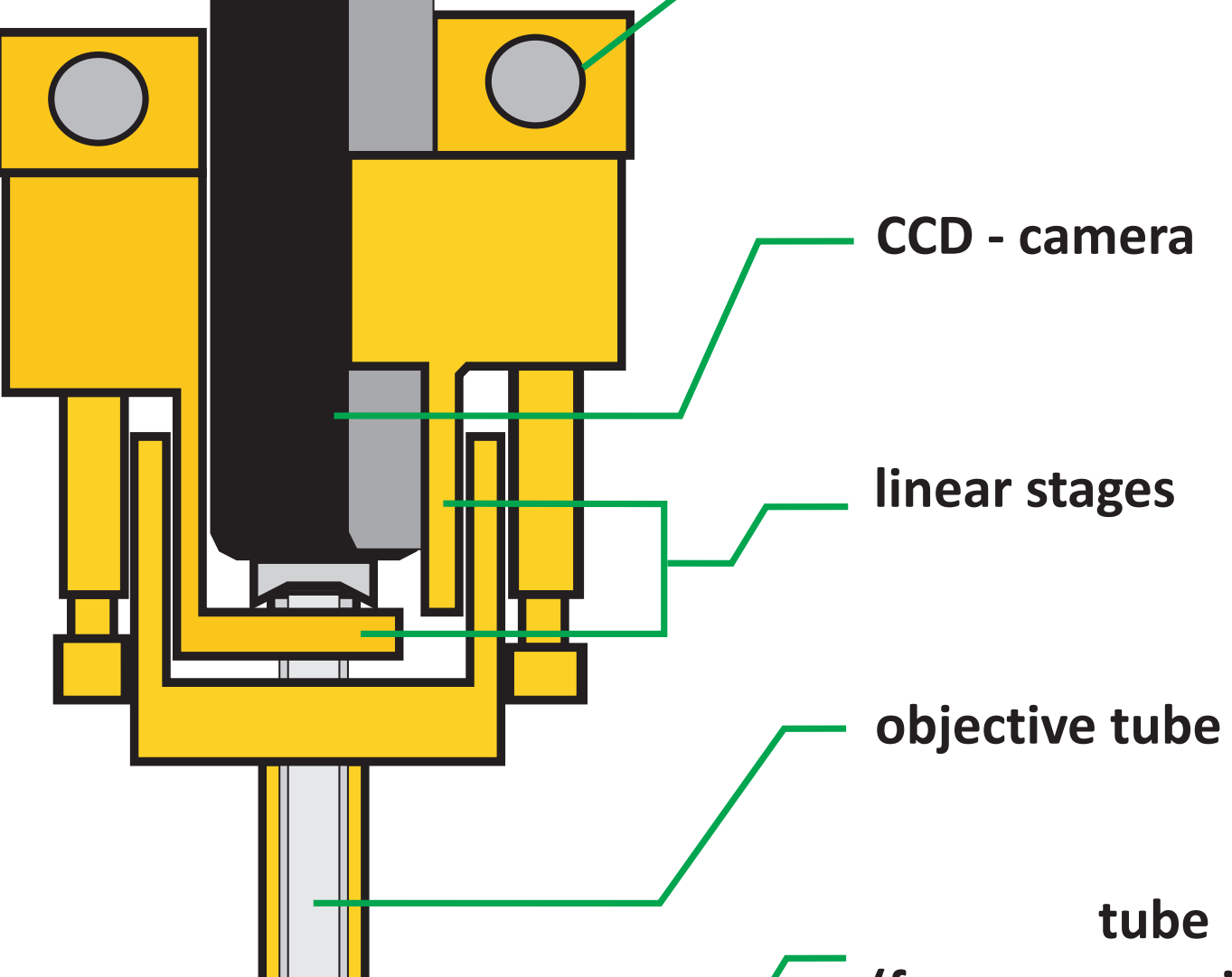

(forms measuring zone)

microscope body

condenser lens

light source

measuring zone

(sterile zone)
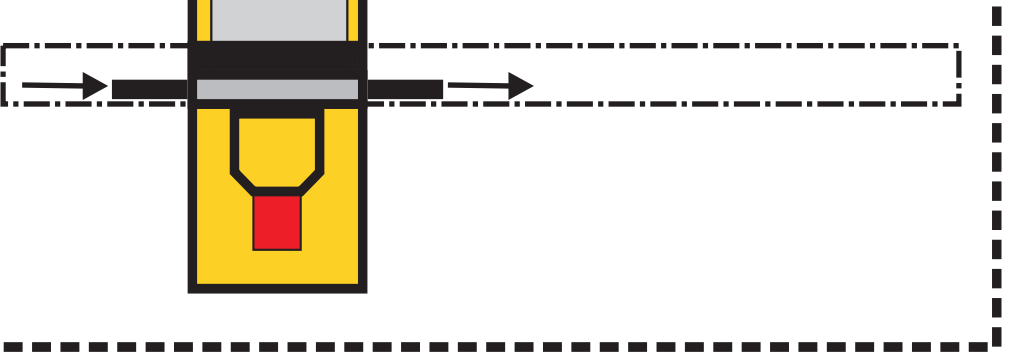
$\searrow$
$\stackrel{0}{0}$
0
$\stackrel{0}{0}$
$\stackrel{0}{\Sigma}$

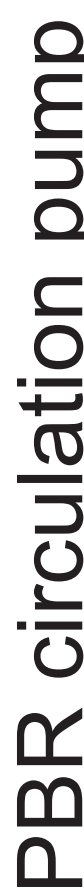

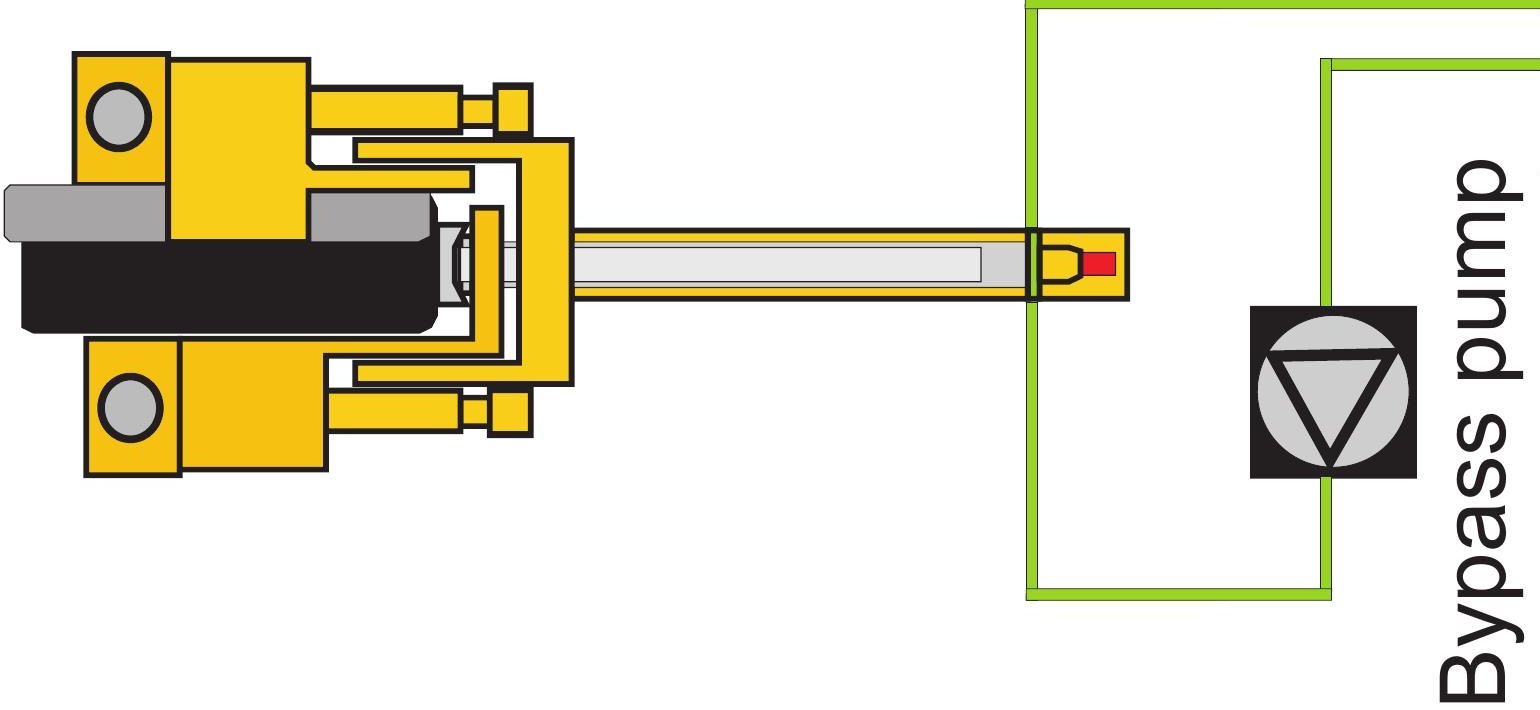




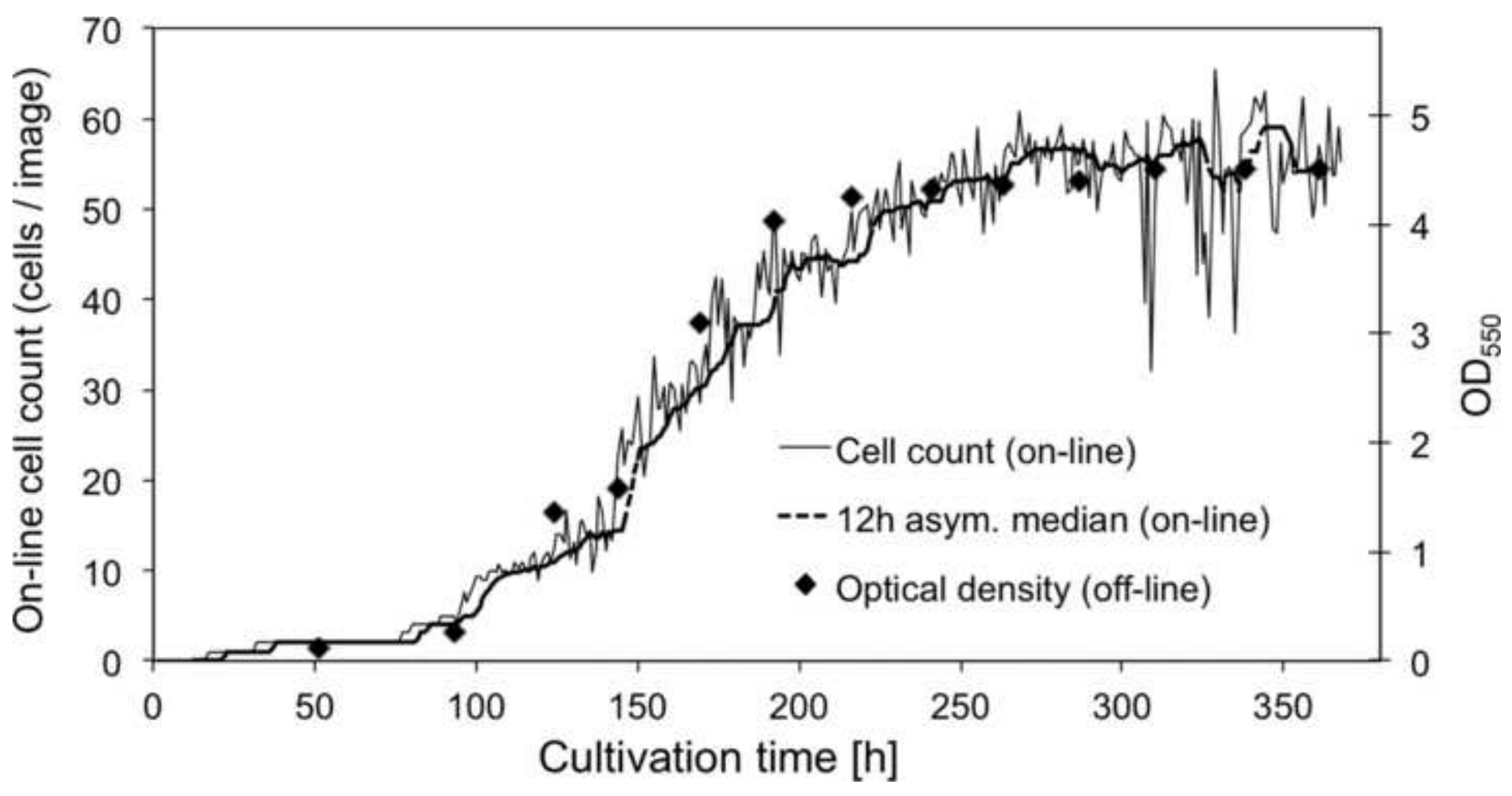




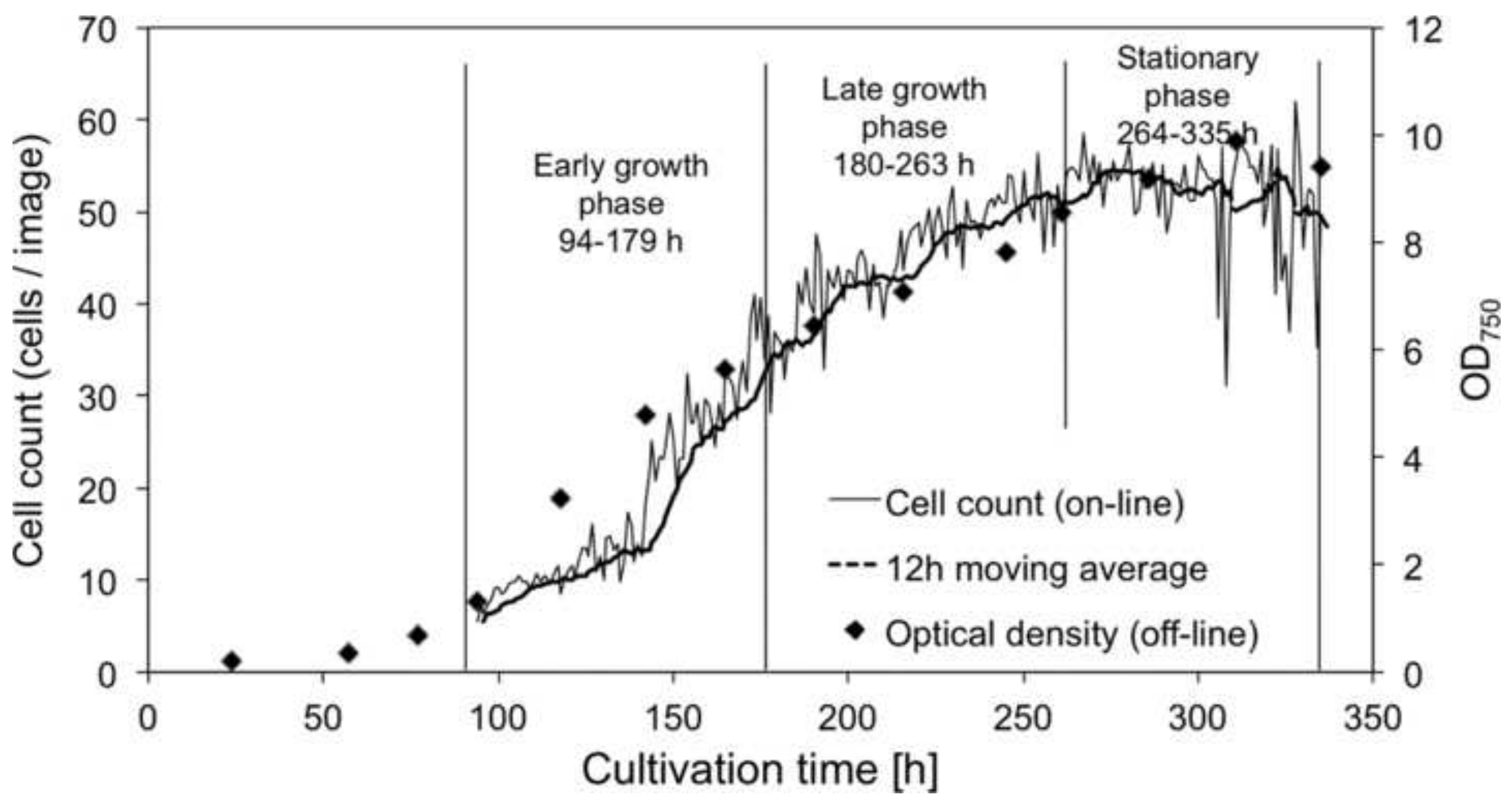




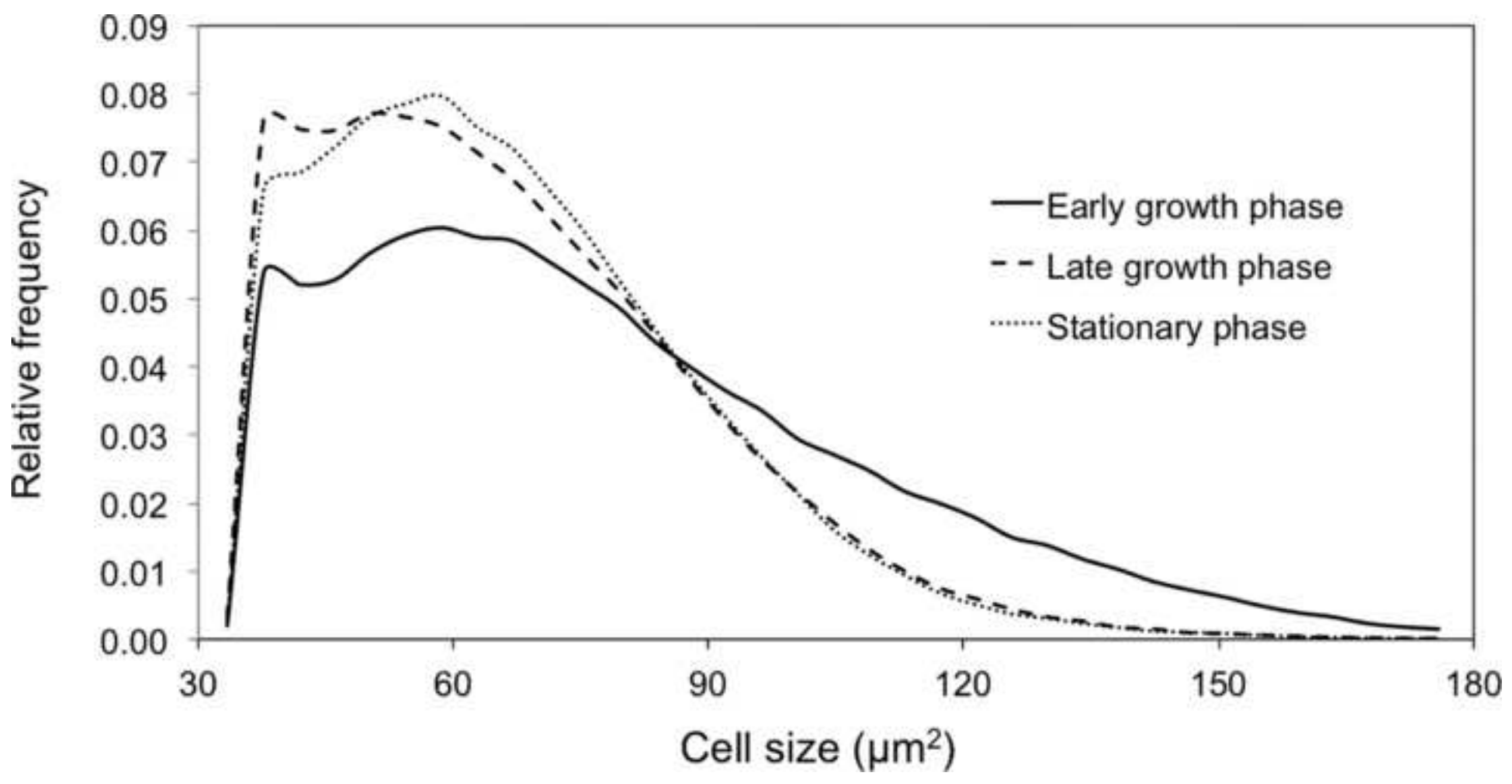




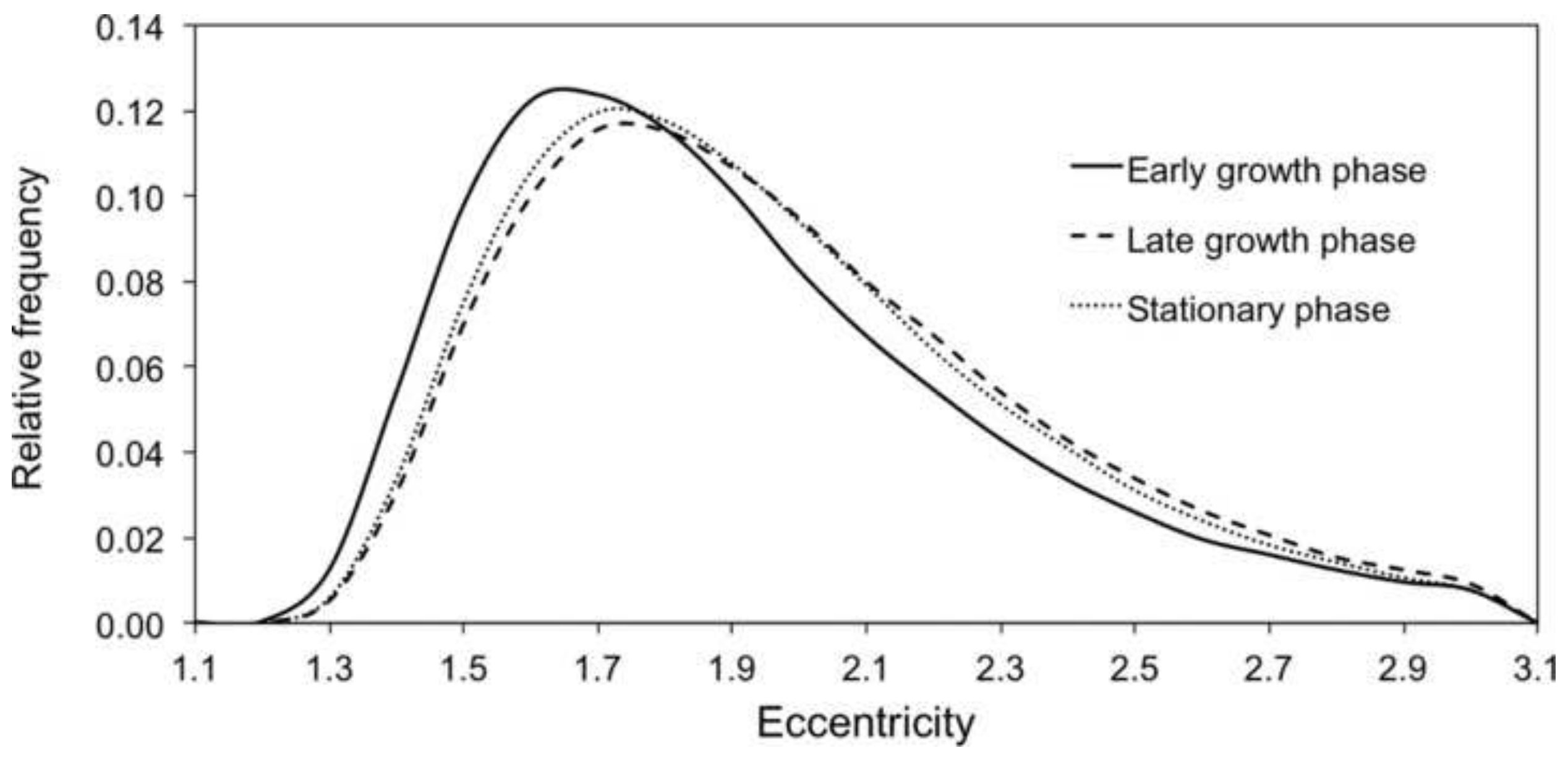


Supplemental Information for Monitoring of microalgal cultivations with on-line, flow-through microscopy Ivo Havlik*, Kenneth F. Reardon, Mehmet Ünal' Patrick Lindner, Andreas Prediger, Alexander Babitzky, Sascha Beutel, and Thomas Scheper

Contents:

Figure S-1. Relative deviations of the ratio of off-line OD to on-line FTM cell counts from the mean of 0.83 computed over an entire cultivation of Chlamydomonas reinhardtii.

Figure S-2. Critical eccentricity function for analysis of Chlorella vulgaris image data.

Figure S-3. Distributions of cell size during different phases of a Chlorella vulgaris cultivation in PBR-2 as measured by the FTM.

Figure S-4. Distributions of morphology in terms of eccentricity during different phases of a Chlorella vulgaris cultivation in PBR-2 as measured by the FTM. 


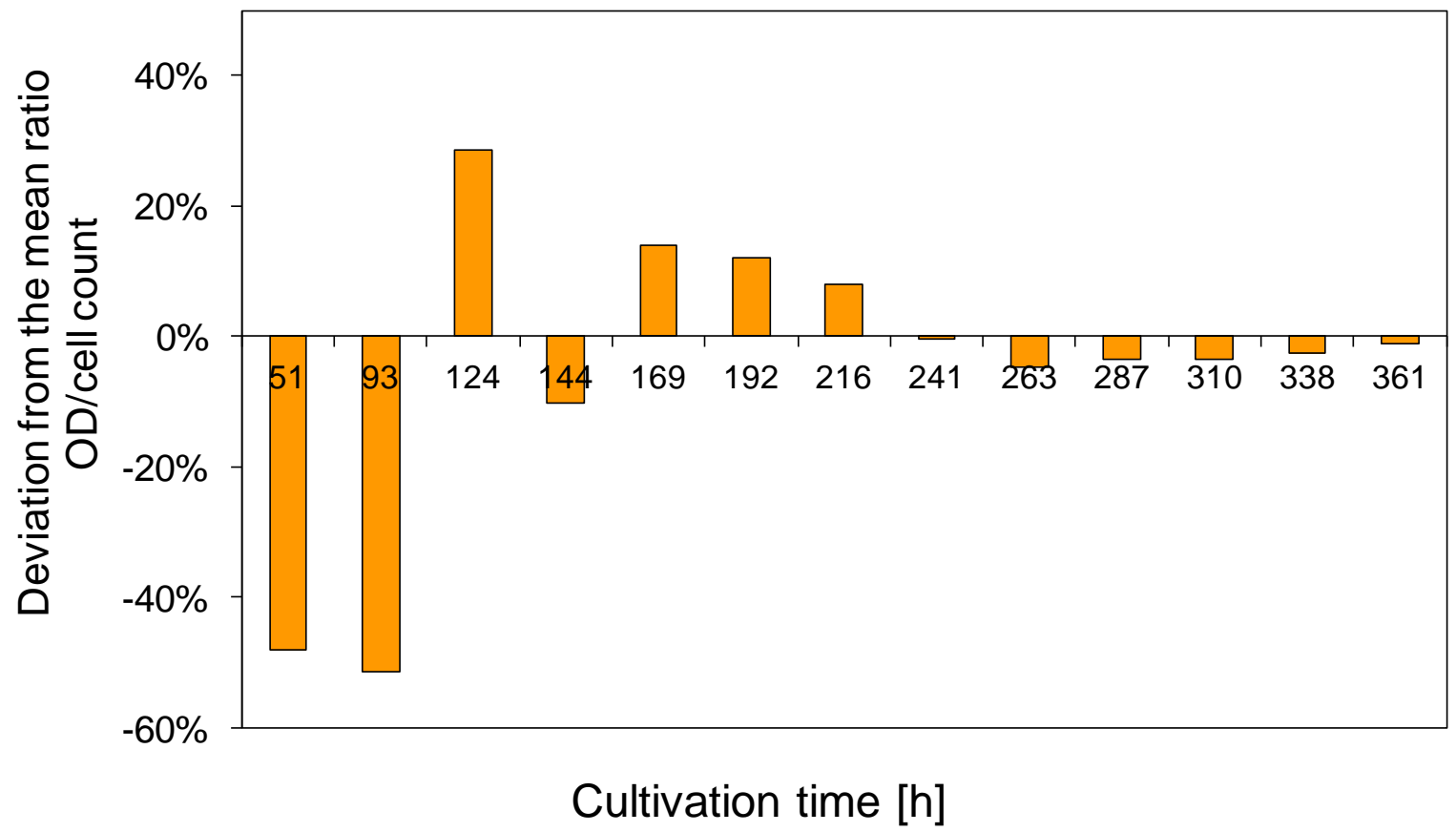

Figure S-1. Relative deviations of the ratio of off-line OD to on-line FTM cell counts from the mean of 0.83 computed over an entire cultivation of Chlamydomonas reinhardtii. 


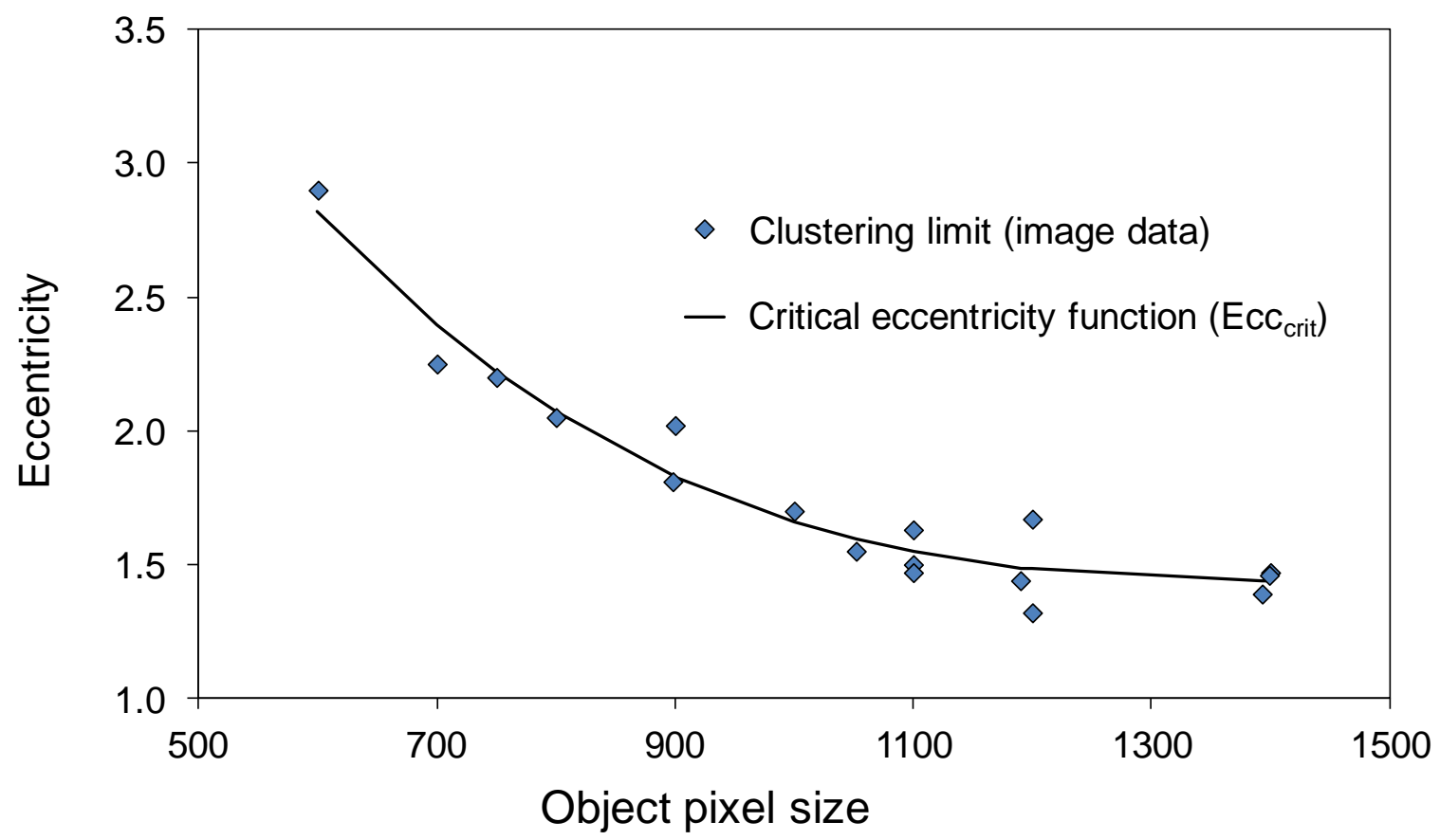

Figure S-2. Critical eccentricity function for analysis of Chlorella vulgaris image data. Manually selected image data (only in the early growth phase) was used to develop a critical eccentricity function for the size range $600-1400$ pixels that serves to distinguish clusters and large single cells. All objects larger than $1400 \mathrm{px}$ are assumed to be clusters, all objects smaller than $600 \mathrm{px}$ are assumed to be single cells, and for objects in the size range $600-1400 \mathrm{px}$, a critical eccentricity is computed. All objects below the critical excentricity (provided by the function) for a given pixel size are assumed to be single cells. Objects classified as clusters can be then removed from further analysis concerning cell size and eccentricity. 


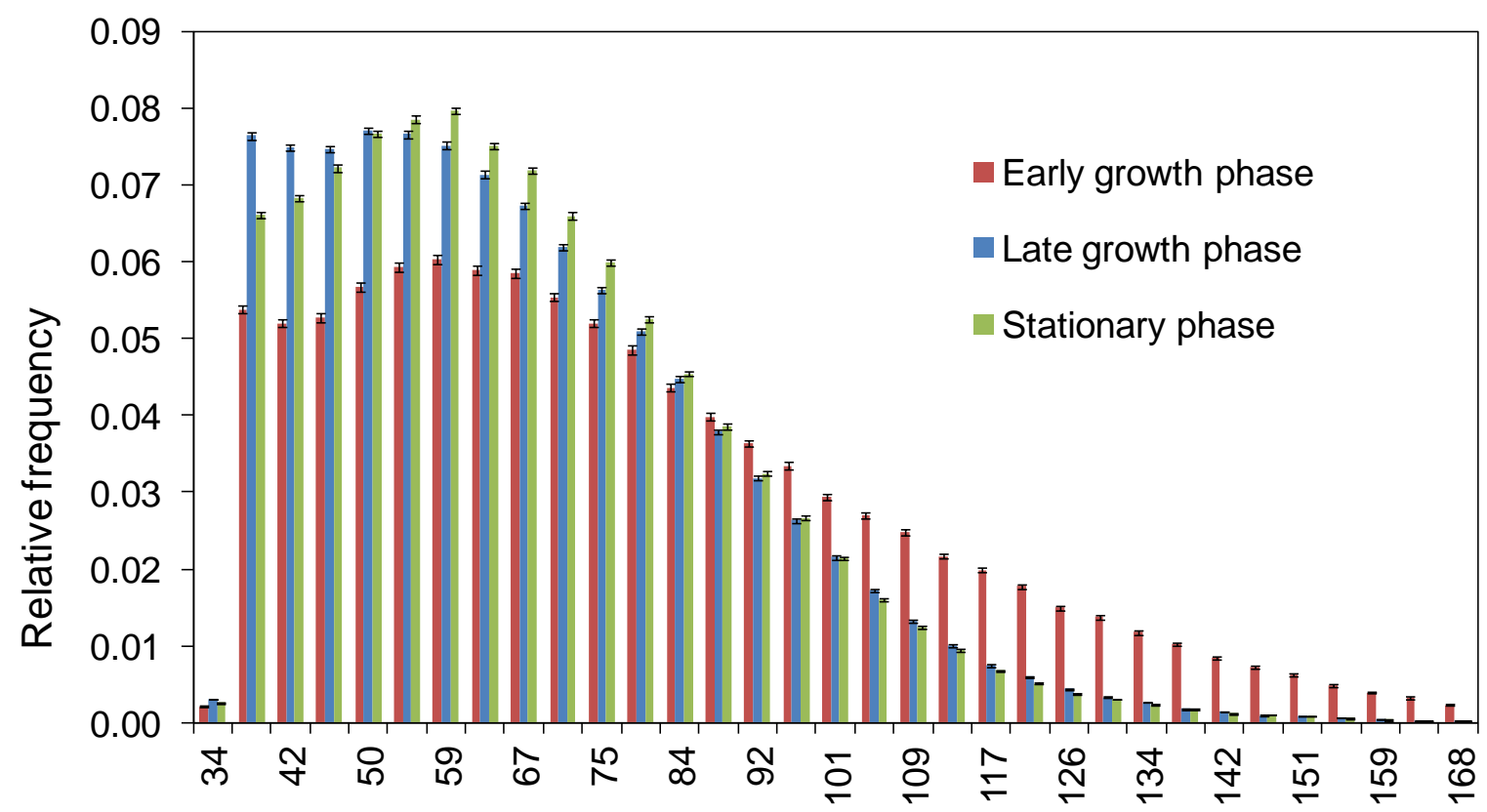

Cell size $\left(\mu \mathrm{m}^{2}\right)$

Figure S-3. Distributions of cell size during different phases of a Chlorella vulgaris cultivation in PBR-2 as measured by the FTM. The early growth phase (94-179 $\mathrm{h}$ ), the late growth phase (180-263 h), and the stationary phase (264-335 h) are shown. Data resulting from cell clusters were removed using the experimentally derived function described in the text. Error bars were calculated for individual histogram bins by assuming Poisson distribution of count error; to normalize for relative frequency, the error in each bin with count $\mathrm{N}$ is equal to $+/-$

$\operatorname{Sqrt}(\mathrm{N}) / \operatorname{Sum}\left(\mathrm{N}_{1} . . \mathrm{N}_{20}\right)$. 


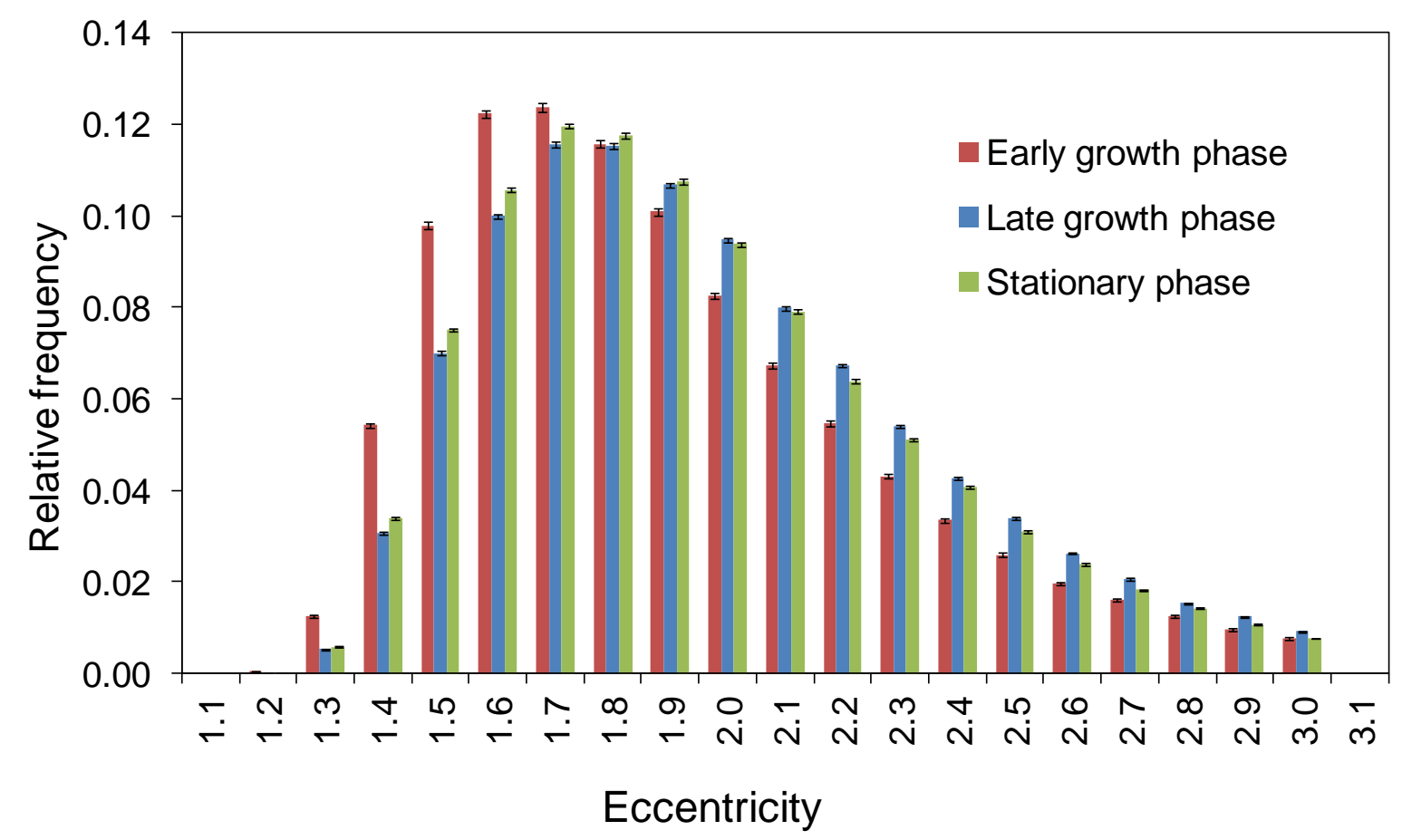

Figure S-4. Distributions of morphology in terms of eccentricity during different phases of a Chlorella vulgaris cultivation in PBR-2 as measured by the FTM. Eccentricity is defined such that a value of 1.0 corresponds to a circle and larger values are ellipses. The early growth phase (94-179 h), the late growth phase (180-263 h), and the stationary phase (264-335 h) are shown. Data resulting from cell clusters were removed using the experimentally derived function described in the text. Error bars were calculated for individual histogram bins by assuming Poisson distribution of count error; to normalize for relative frequency, the error in each bin with count $\mathrm{N}$ is equal to $+/-\operatorname{Sqrt}(\mathrm{N}) / \operatorname{Sum}\left(\mathrm{N}_{1} . . \mathrm{N}_{20}\right)$. 